\title{
Modulation Instability of Two-Dimensional Dipolar Bose-Einstein Condensate in a Deep Optical Lattice
}

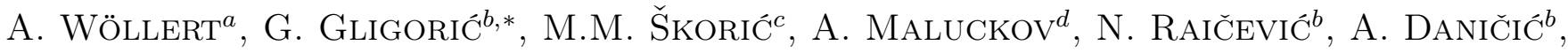 \\ AND LJ. HADŽIEVSKI ${ }^{b}$ \\ ${ }^{a}$ Faculty of Physics, University of Göttingen, Germany \\ ${ }^{b}$ Vinča-Institute of Physics, Belgrade, Serbia \\ ${ }^{c}$ National Instutute for Fusion Science, Tokio, Japan \\ ${ }^{d}$ Faculty of Sciences and Mathematics, Niš, Serbia
}

\begin{abstract}
We study the stability of the continuous waves in the pancake shaped dipolar Bose-Einstein condensate trapped in the strong optical lattice potential with the coexisting local (the short-range $s$-wave) interaction and nonlocal (the dipole-dipole) interactions between the condensate atoms. The system is modeled by two two-dimensional discrete models derived from the Gross-Pitaevskii equation accounting the dipole-dipole interactions: discrete nonlinear Schrödinger equation with cubic nonlinearity and nonpolynomial Schrödinger equation. The corresponding dispersion relations are calculated analytically and the regions of the modulation instability in the parametric space are summarized into the stability diagrams.
\end{abstract}

PACS numbers: 03.75.Lm, 05.45.Yv

\section{Introduction}

The modulation instability (MI) is a general characteristic of wave propagation in nonlinear dispersive media and has been studied in diverse fields as fluid dynamics [1], nonlinear optics [2], plasma physics [3], matter waves [4], etc. It is associated with a process in which weak perturbations to the steady state increase exponentially as a result of interplay between the nonlinearity and the group-velocity dispersion. The modulation instability is closely related to the localized mode formation, occurring in the same parameter region where localized soliton-like structures have been observed.

In this paper we consider MI of the continuous-wave solution (CW) as a precursor for localized soliton-like structures existence in pancake shaped dipolar Bose-Einstein condensates (BEC) in a very deep optical lattice [5]. The presence of a deep optical lattice gives possibility to model the BEC with two discrete versions of the two-dimensional Gross-Pitaevskii equation (GPE) accounting the dipole-dipole interactions (DD), discrete nonlinear Schrödinger equation (NLSE) with usual cubic nonlinearity [5] and more accurate discrete nonpolynomial Schrödinger equation (NPSE) [6]. A noteworthy feature of the discrete NPSE is that it may account for the onset of collapse which is experimentally found in real 3D BEC with attractive contact interaction.

The analysis of the modulation instability is presented for both discrete models and for all combinations of the

\footnotetext{
* corresponding author; e-mail: goran79@vinca.rs
}

contact and DD interaction types: attractive and repulsive contact, isotropic and anisotropic DD interaction. The corresponding dispersion equations are derived analytically and regions of the MI are summarized into the stability diagrams.

\section{Modulation instability}

Discrete 2D NPSE with dipole-dipole interaction can be written in the following form:

$$
\begin{aligned}
& \mathrm{i} \frac{\partial \phi_{m, n}}{\partial t}=-\frac{C}{2} \\
& \quad \times\left(\phi_{m+1, n}+\phi_{m-1, n}+\phi_{m, n+1}+\phi_{m, n-1}-4 \phi_{m, n}\right) \\
& \quad+\left[\frac{\aleph\left|\phi_{m, n}\right|^{2}}{\eta_{m, n}}+\frac{1}{2}\left(\frac{1}{\eta_{m, n}^{2}}+\eta_{m, n}^{2}\right)+\Gamma \sum_{m^{\prime}, n^{\prime}}\right. \\
& \left.\quad \frac{1-3 \cos ^{2} \theta_{m^{\prime}, n^{\prime}}}{\left|\left(m-m^{\prime}\right)^{2}+\left(n-n^{\prime}\right)^{2}\right|^{3 / 2}}\left|\phi_{m^{\prime}, n^{\prime}}\right|^{2}\right] \phi_{m, n}, \\
& \eta_{m, n}^{4}=1+\aleph\left|\phi_{m, n}\right|^{2} \eta_{m, n},
\end{aligned}
$$

where the parameter $C$ is the coupling constant, $\aleph=-1$ corresponds to the attractive and $\aleph=1$ to repulsive contact interaction, $\Gamma$ is the ratio between the strength of the dipole-dipole and contact interaction and angle $\theta_{m^{\prime}, n^{\prime}}$ is $\pi / 2$ or 0 for polarization of dipoles along the axial $(z)$ direction or in the perpendicular plane (we choose polarization along $x$ axis), respectively. In the former case DD interaction is isotropic and in the later anisotropic. The 
first equation in system (1) is discrete version of the evolution equation for the transverse wave function [6] and the second equation in system (1) determines the axial width of the wave function [6]. Equation (1) can be reduced to the discrete 2D GPE with cubic nonlinearity in a low density limit $\left|\phi_{m, n}\right| \ll 1$, when the axial width of the wave function becomes fixed, $\eta_{m, n}=\eta=1$. The stationary CW solution of Eq. (1) has a form $\phi_{m, n}=\phi \mathrm{e}^{-\mathrm{i} \mu t}$ where parameter $\mu$ is the propagation constant or chemical potential in the context of BEC. Substituting CW solution in Eq. (1) we obtain the relation between $\mu$ and amplitude $\phi$ according to which the CW solutions exist only for certain range of the parameters $\mu$ and $\Gamma$ satisfying

$$
\phi^{2}=\frac{\mu-\left(1+\eta^{4}\right) /\left(2 \eta^{2}\right)}{\aleph / \eta+\Gamma \Sigma_{x 0, z 0}}>0, \quad \eta>0,
$$

where the sums depending on the type of the dipoledipole interaction are given with the expressions (5) or $(6)$.

In order to clarify the problem of the MI of the CW solution we follow the standard procedure and look for solutions of Eq. (1) in form $\phi_{m, n}=\left(\phi+\delta u_{m, n}\right) \mathrm{e}^{-\mathrm{i} \mu t}$ where $\delta u_{m, n}=a_{m, n}+\mathrm{i} b_{m, n}$ represents small complex perturbations which modulate the CW solution. Substituting perturbed solution into Eq. (1) and linearizing the resulting equations, we obtain a system of two coupled linear equations for $a_{m, n}$ and $b_{m, n}$. Looking for solutions of the perturbation functions in a form of plane waves $\sim \exp (\Omega t+\mathrm{i} p m+\mathrm{i} q n)$, where $p$ and $q$ are wave numbers in lattice directions $m$ and $n$, we obtain the dispersion relations.

In the case of the 2D GPE with cubic contact and dipole-dipole interaction the dispersion relation has a form

$$
\begin{gathered}
\Omega_{z, x}^{2}=-4 C^{2}\left[\sin ^{2}\left(\frac{p}{2}\right)+\sin ^{2}\left(\frac{q}{2}\right)\right]^{2} \\
\times\left[1+\frac{\phi^{2}\left(\Gamma \Sigma_{z, x}+\aleph\right)}{C\left(\sin ^{2}\left(\frac{p}{2}\right)+\sin ^{2}\left(\frac{q}{2}\right)\right)}\right],
\end{gathered}
$$

while the dispersion relation for 2D NPSE model reads

$$
\begin{aligned}
& \Omega_{z, x}^{2}=-4 C^{2}\left(\sin ^{2}\left(\frac{p}{2}\right)+\sin ^{2}\left(\frac{q}{2}\right)\right)^{2} \\
& \quad \times\left[1+\Gamma \phi^{2} \frac{\Sigma_{z, x}-\Sigma_{z 0, x 0}}{2 C\left(\sin ^{2}\left(\frac{p}{2}\right)+\sin ^{2}\left(\frac{q}{2}\right)\right)}\right] \\
& \quad \times\left[1+\phi \frac{\left(v \phi+\alpha \phi^{2}\right)\left(\frac{3}{2} v \phi^{2}+2 \aleph\right)}{\left(1+\aleph v \phi^{2}\right)^{3 / 2}\left(\sin ^{2}\left(\frac{p}{2}\right)+\sin ^{2}\left(\frac{q}{2}\right)\right)}\right. \\
& \left.+\Gamma \frac{\phi^{2}\left(3 \Sigma_{z, x}-\Sigma_{z 0, x 0}\right)}{2 C\left(\sin ^{2}\left(\frac{p}{2}\right)+\sin ^{2}\left(\frac{q}{2}\right)\right)}\right],
\end{aligned}
$$

where parameters $v$ and $\alpha$ are defined with

$$
v=\frac{1}{2}\left[\aleph \sqrt{2(A+B)}+\sqrt{-2(A+B)+\frac{2 \phi^{2}}{\sqrt{2(A+B)}}}\right],
$$

$$
\begin{aligned}
\alpha & =\frac{1}{2}\left\{\frac{\frac{\phi}{\sqrt{2(A+B)}}\left[1-\frac{\phi^{4}(A-B)}{48(A+B)}\right]-\frac{\phi^{3}}{24}(A-B)}{\sqrt{-2(A+B)+2 \phi^{2} / \sqrt{2(A+B)}}}\right. \\
& \left.+\aleph \frac{\phi^{3}(A-B)}{24 \sqrt{2(A+B)}}\right\} \\
A & =\sqrt[3]{\left(\phi^{4} / 4^{2}\right)+\sqrt{\left(\phi^{8} / 4^{4}\right)+(1 / 3)^{3}}}, \\
B & =\sqrt[3]{\left(\phi^{4} / 4^{2}\right)-\sqrt{\left(\phi^{8} / 4^{4}\right)+(1 / 3)^{3}}} .
\end{aligned}
$$

The sums included in Eqs. (2)-(4) for the isotropic DD interaction are

$$
\begin{aligned}
& \Sigma_{z}=\sum_{m^{\prime}, n^{\prime}} \frac{\mathrm{e}^{\mathrm{i}\left[p\left(n-n^{\prime}\right)+q\left(m-m^{\prime}\right)\right]}}{\left|\left(m-m^{\prime}\right)^{2}+\left(n-n^{\prime}\right)^{2}\right|^{3 / 2}}, \\
& \Sigma_{z 0}=\sum_{m^{\prime}, n^{\prime}} \frac{1}{\left|\left(m-m^{\prime}\right)^{2}+\left(n-n^{\prime}\right)^{2}\right|^{3 / 2}},
\end{aligned}
$$

while for the anisotropic DD interaction are

$$
\begin{aligned}
& \Sigma_{x}=\sum_{m^{\prime}, n^{\prime}} \\
& \frac{\mathrm{e}^{\mathrm{i}\left[p\left(n-n^{\prime}\right)+q\left(m-m^{\prime}\right)\right]\left[\left(n-n^{\prime}\right)^{2}-2\left(m-m^{\prime}\right)^{2}\right]}}{\left|\left(m-m^{\prime}\right)^{2}+\left(n-n^{\prime}\right)^{2}\right|^{5 / 2}}, \\
& \Sigma_{x 0}=\sum_{m^{\prime}, n^{\prime}} \frac{\left(n-n^{\prime}\right)^{2}-2\left(m-m^{\prime}\right)^{2}}{\left|\left(m-m^{\prime}\right)^{2}+\left(n-n^{\prime}\right)^{2}\right|^{5 / 2}} .
\end{aligned}
$$

\section{Results and discussion}

The dispersion relations (3) and (4) enable calculating the borders of the MI region in the parametric space $(\Gamma, \mu)$ which correspond to the regions with positive $\Omega^{2}$ indicating exponential growth of the introduced perturbations and MI of the initial CW solution. In these regions the existence of new localized solutions are expected. The corresponding stability diagrams are displayed in Figs. 1 and 2 for discrete 2D NPSE, with attractive and repulsive contact interaction, respectively. For any point into the parametric space, the appearance of $\Omega^{2}>0$ at least for one pair of $(p, q)$ was the sufficient condition to identify MI.

The stability diagrams (Fig. 1) clearly show that the presence of the DD interactions in the BEC with attractive contact interaction extends regions of $\mathrm{MI}$ and indicates extension of the existence region for the localized solitary structures. The stability diagrams (Fig. 2) show that the increase of the intensity of the non-local dipoledipole interaction leads to the appearance of the MI regions for BEC with repulsive contact interaction. This result indicates possibility for existence of the unstaggered localized structures in BEC with repulsive contact 


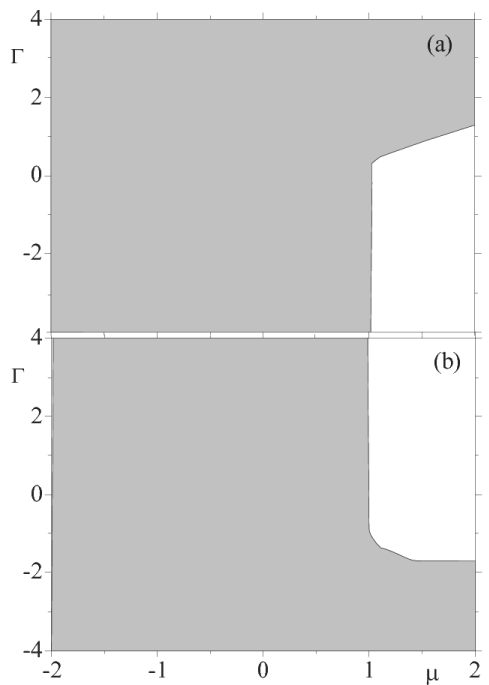

Fig. 1. The 2D NPSE with $C=0.8$ and attractive contact interaction. The gray color stands for the MI region and white for the region with stable CW solutions. Plot (a) corresponds to the isotropic DD and (b) to the anisotropic DD interaction.

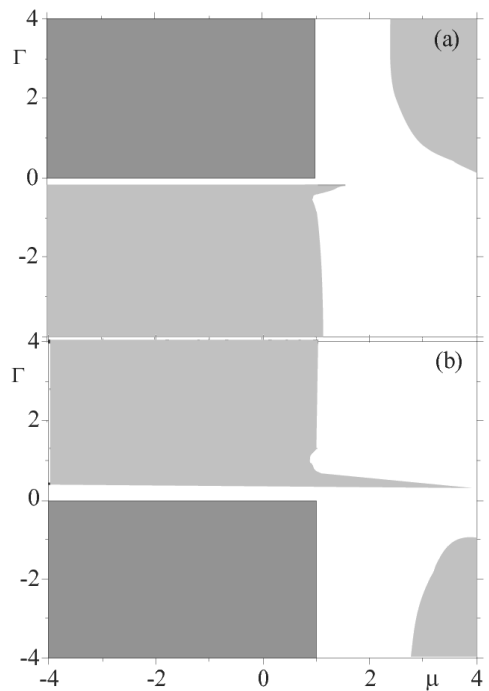

Fig. 2. The 2D NPSE with $C=0.8$ and repulsive contact interaction. The light gray color stands for the MI region, white for the stable $\mathrm{CW}$ and dark gray denotes the regions where CW solutions cannot exist [the conditions written in relation (2) are not simultaneously satisfied]. Plot (a) corresponds to the isotropic DD and (b) to the anisotropic DD interaction.

interaction as were found in literature $[7,8]$. In addition, the results show that the influence of the dipole orientation on the stability of the $\mathrm{CW}$ solutions reflects only to the opening of instability regions for different sets of system parameters.
Qualitatively the same results considering development of the MI of the CW solutions are obtained for the pancake BEC modeled by the discrete 2D NLSE with cubic contact (local) and DD (non-local) interaction. Only the corresponding graphs are shifted with respect to $\mu\left(\mu_{\mathrm{gpe}} \sim \mu_{\mathrm{npse}}-1\right)$.

\section{Conclusion}

The Bose-Einstein condensates with different atomic species and various trapping geometries are nowadays in the focus of the researchers of many experimental groups all over the world. One of the main streamlines is generation of the localized patterns. In this paper we studied such system consisting of the pancake BEC with dipolar atoms in a very deep optical lattice within the framework of two 2D discrete models: NLSE and NPSE. Both of them include the same non-local term describing the DD interaction. The results show that both models produce qualitatively the same results considering development of the MI. The presence of the DD interaction of both types - isotropic and anisotropic - extends the region of $\mathrm{MI}$ in the parametric space for BEC with attractive contact nonlinearity. Also, the increase of the attractive DD interaction produces $\mathrm{MI}$ for the BEC with a repulsive contact interaction. These results indicate that the DD interaction extends the parameter region of the localized structures existence in the BAC with attractive contact interaction, but in the repulsive BEC creates regions of existence of the localized structures which are not present without support of the strong attractive DD interaction. The main findings are consistent with the results presented in literature $[5,7,8]$.

\section{Acknowledgments}

G.G., A.M., M.M.Š. and Lj.H. acknowledge support from the Ministry of Science, Serbia, Project 141034.

\section{References}

[1] T.J. Benjamin, J.E. Feir, J. Fluid Mech. 27, 417 (1967).

[2] L.A. Ostrovskiy, Zh. Eksp. Teor. Fiz. 51, 1189 (1966).

[3] A. Hasegawa, Plasma Instabilities and Nonlinear Effects, Springer-Verlag, Heidelberg 1975.

[4] V.M. Lashkin, Phys. Rev. A 75, 043607 (2007).

[5] P.G. Kevrekidis, K.Ø. Rasmussen, A.R. Bishop, Phys. Rev. E 61, 2006 (2000).

[6] L. Salasnich, B.A. Malomed, Phys. Rev. A 79, 053620 (2009).

[7] P. Pedri, L. Santos, Phys. Rev. Lett. 95, 200404 (2005).

[8] I. Tikhonenkov, B.A. Malomed, A. Vardi, Phys. Rev. Lett. 100, 090406 (2008). 